\title{
Pump-Probe X-ray Holographic Imaging of Dynamic Magnetization Processes Down to the Femtosecond Timescale
}

\author{
Stefan Eisebitt ${ }^{1,2,3}$
}

1. Institut für Optik und Atomare Physik, Technische Universität Berlin, 10623 Berlin, Germany

2. Div. of Synchrotron Radiation Research, Lund University, SE-221 00 Lund, Sweden

3. Joint Research Group Functional Nanomaterials, Helmholtz Zentrum Berlin, 14109 Berlin, Germany

X-ray holography as an approach for nanoscale imaging is experiencing a boost facilitated by the possibility to realize Fourier transform holography geometries via lithographic masks [1] in conjunction the increasing availability of x-ray sources with appreciable coherent photon flux, namely: accelerator based free-electron lasers and $3^{\text {rd }}$ generation synchrotron sources as well as laser driven sources such as based on high harmonic generation (HHG). All these sources are intrinsically pulsed with pulse durations ranging from picoseconds to attoseconds. With the ability to excite resonant electronic transitions from core to valence levels and the resulting detailed spectroscopic information which can be used as a contrast mechanism for imaging, time resolved spectro-holographic studies with resolution down to nanometers and femtoseconds have become possible. In particular, studies of magnetization dynamics on the nanoscale are feasible via x-ray holography exploiting $\mathrm{x}$-ray magnetic circular dichroism (XMCD) contrast [1,2]. In fact, so far the only spatially resolved magnetization maps recorded at free-electron $\mathrm{x}$-ray lasers were recorded via holography, exploiting the high coherent photon number per pulse for fs single shot imaging [3]. Here, on behalf of the authors in Refs [4] and [5], I report on recent advances in combining X-ray holography with XMCD contrast for the study of dynamic magnetization rearrangements on the nanoscale with temporal resolution in the ps and fs regime via repetitive pump-probe experiments. The experiments are carried out via mask-based Fourier Transform x-ray holography (FTH) in transmission as described in Refs.[1,6]

Magnetic field induced dynamic in a thin magnetic film with perpendicular anisotropy patterned in a $550 \mathrm{~nm}$ disk was studied at BESSY II in single bunch mode using soft x-rays at the Co $\mathrm{L}_{3}$ edge for XMCD contrast [4]. The magnetic sample and holographic mask were monolithically integrated, together with a micro coil allowing to apply a pulsed magnetic field via a pulsed current.[7] The rigid integration of the sample with the critical imaging components (i.e. object aperture and reference aperture) as well as the micro coil leads to insensitivity against vibration and drift, resulting in the very high real space tracking accuracy of about $3 \mathrm{~nm}$ shown below. The multilayer film with composition $\mathrm{Pt}(2) /\left[\mathrm{Co}_{68} \mathrm{~B}_{32}(0.4) / \mathrm{Pt}(0.7)\right]_{30} / \mathrm{Pt}(1.3)$ (thickness in $\mathrm{nm}$ ) was developed for low pinning center density [8] to facilitate the observation of deterministic domain wall motion not dominated by stochastic movements from one pinning site to the next. In Fig. 1(left) we show a SEM micrograph of the sample structure. A static magnetic field was applied via an in vacuum quadrupole magnet in order to bring the magnetic domain configuration within the disk to a point where a bubble domain exists. Such a bubble has the topology of a skyrmion (with skyrmion number 1) and the study of its dynamics when displaced from its equilibrium position was the goal of this work. The displacement from the equilibrium position was accomplished via a bipolar magnetic field pulses, which reproducibly nucleate and subsequently annihilate an additional magnetic domain. The dipolar interactions with this domain lead to the skyrmion displacement. In Fig. 1(right) we present the skyrmion trajectory, recorded with 50 ps (sigma) temporal resolution. Note that while the spatial resolution in the hologram reconstructions is $40 \mathrm{~nm}$, the tracking accuracy is about $3 \mathrm{~nm}$ (depending on data point), reflecting the accuracy with which we can 
determine the center of magnetization of the skyrmion based on the repetitive pump-probe measurements with good statistics. This is possible due to the extraordinary stability and drift insensitivity of monolithic FTH holography. The analysis of the trajectory allowed us to conclude that the skyrmion has to be described with an inertial mass, and to determine the lower limit of this mass [4].

Results from a pump probe FTH experiment in the context of ultrafast optical demagnetization are shown in Fig. 2. [5] Here, an infrared laser pulse was used to excite a perpendicular anisotropy multilayer film with composition $\mathrm{Pd}(2)[\mathrm{Co}(0.4) \mathrm{Pd}(0.2)]_{20} \mathrm{Al}(3)$. The optical excitation was localized within the elliptical object hole (defining the field of view) via suitable reflection from the elliptical aperture wall and interference. This region is clearly visible at a pump-probe delay of $600 \mathrm{fs}$ at the top of the elliptical field of view, as the magnetic domain contrast, representing up/down magnetized regions, is washed out due to transient demagnetization. At different delays, a spatio-temporal evolution of this region is observed. These non-local changes in the demagnetization region are consistent with mechanisms including ultrafast transport of spin-polarized electrons during the demagnetization process. This experiment was carried out at the DiProI endstation at the FERMI free-electron laser at the Co M edge. Note that despite the large wavelength of $20.8 \mathrm{~nm}$, high resolution imaging is possible [2], which is of particular interest for use with coherent laboratory sources in the near future.
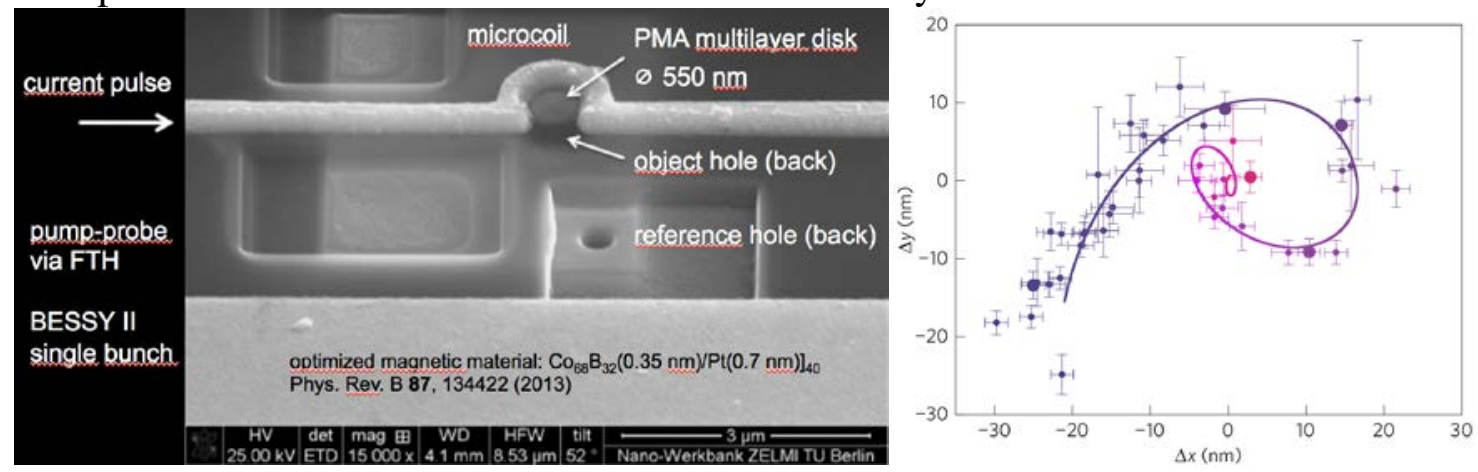

Figure 1. Left: Sample for FTH after magnetic field pump. Right: Skyrmion trajectory determined from pump-probe FTH. Time scale ranges from zero (blue ) to 14 ns (red). [4]

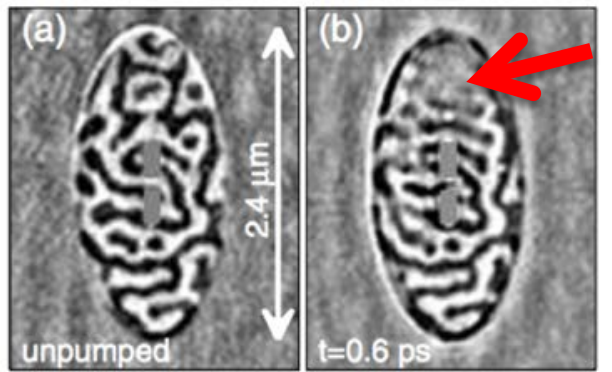

Figure 2. FTH imaging of optically induced demagnetization. Left: unpumped sample, elliptical field of view. Black and white contrast indicates magnetization pointing up/down. Right: local pump at top (red arrow), imaged at 600 fs delay [5]

\section{References:}

[1] S. Eisebitt et al., Nature 432, 885 (2004).

[2] S. Schaffert et al., New J. Phys. 15, 093042 (2013).

[3] T.H. Wang et al. Phys. Rev. Lett. 108, 267403 (2012).

[4] F. Büttner et al, Nature Physics, advance online publication DOI: 10.1038/nphys3234 (2015)

[5] C. von Korff Schmising et al., Phys. Rev. Lett. 112, 217203 (2014)

[6] W.F. Schlotter et al., Appl. Phys. Lett. 89 (2006).

[7] F. Büttner et al., Opt. Express 21, 30563 (2013).

[8] F. Büttner et al., Phys. Rev. B 87 (2013). 